\title{
Solvation force, structure and thermodynamics of fluids confined in geometrically rough pores
}

\author{
Chandana Ghatak and K. G. Ayappa ${ }^{\text {a) }}$ \\ Department of Chemical Engineering, Indian Institute of Science, Bangalore 560012, India
}

\begin{abstract}
The effect of periodic surface roughness on the behavior of confined soft sphere fluids is investigated using grand canonical Monte Carlo simulations. Rough pores are constructed by taking the prototypical slit-shaped pore and introducing unidirectional sinusoidal undulations on one wall. For the above geometry our study reveals that the solvation force response can be phase shifted in a controlled manner by varying the amplitude of roughness. At a fixed amplitude of roughness, $a$, the solvation force for pores with structured walls was relatively insensitive to the wavelength of the undulation, $\lambda$ for $2.3<\lambda / \sigma_{f f}<7$, where $\sigma_{f f}$ is the Lennard-Jones diameter of the confined fluid. This was not the case for smooth walled pores, where the solvation force response was found to be sensitive to the wavelength, for $\lambda / \sigma_{f f}<7.0$ and amplitudes of roughness, $a / \sigma_{f f} \geqslant 0.5$. The predictions of the superposition approximation, where the solvation force response for the rough pores is deduced from the solvation force response of the slit-shaped pores, was in excellent agreement with simulation results for the structured pores and for $\lambda / \sigma_{f f} \geqslant 7$ in the case of smooth walled pores. Grand potential computations illustrate that interactions between the walls of the pore can alter the pore width corresponding to the thermodynamically stable state, with wall-wall interactions playing an important role at smaller pore widths and higher amplitudes of roughness.
\end{abstract}

\section{INTRODUCTION}

Confined nanoscale fluids occur in technologically important processes such as boundary lubrication, adhesion, adsorption, and wetting. Common to these processes is the presence of fluid films that are under the strong influence of confining walls, resulting in fluids that are inhomogeneous. Forces on molecularly thin films have been investigated using the surface force apparatus (SFA) and its variants which measure in addition to shear forces, the solvation force which is the normal force exerted by the fluid on the confining walls. ${ }^{1-4}$ The oscillatory nature of the solvation force observed in surface force experiments is due to the formation and disruption of fluid layers as the degree of confinement is varied. Molecular simulation techniques have been widely used to investigate the thermodynamics of confined fluids in slit-shaped pores, ${ }^{5-12}$ and in particular have aided in understanding the relationship between fluid structure and solvation force at the molecular level. Until recently, these molecular simulation studies have been restricted to fluids confined between walls that represent a particular crystallographic plane of infinite extent. The walls are either smooth (structureless) or structured depending on the form of the fluid-wall interaction potential. In a structured pore, the walls consist of discrete atoms which represent periodic roughness on the atomic scale. Molecular simulations using structured walls are used to demonstrate stick-slip and epitaxial freezing of confined fluids. ${ }^{13}$ Most engineering surfaces are rough either on an atomic and/or higher scales, and solid-solid contact normally occurs at asperities. ${ }^{14}$ Rough-

a)Electronic mail: ayappa@chemeng.iisc.ernet.in ness is known to effect adhesion, ${ }^{15}$ wetting, ${ }^{16}$ friction, and wear. ${ }^{17}$ Understanding the behavior of fluid films on rough surfaces is important if our present knowledge of confined fluid physics is to be extended to more realistic situations.

SFA experiments involving mica surface that have been coated with long chain molecules enable one to probe forces between surfaces that are no longer atomically smooth, at least on the preexisiting scale of the mica surface. In order to elucidate the effects of roughness, experiments were carried out by either adsorbing cetyltrimethylammoniumbromide (CTAB) from solution or depositing Langmuir-Blodgett films of dioctadecyldimethylammoniumbromide (DOAB) on mica surfaces. ${ }^{18}$ CTAB exhibited a complete smearing out of oscillations and DOAB showed a reduced range of oscillations. Their results indicate that the interactions of fluids between rough surfaces may be adequately described by a Lifshitz theory. Recent SFA experiments illustrate that the traditional experimental procedure of cutting mica with hot platinum wire results in nanoparticle deposition on the mica sheets. ${ }^{19,20}$ Although the coverage of nanoparticles is shown to be less than $1 \%$, force curves obtained in the absence of these nanoparticles have been shown to have larger oscillations extending up to ten fluid layers. ${ }^{20}$ Clearly roughness, albeit small, caused by these nanoparticles is seen to quantitatively alter the structure and force response therein of the confined fluid.

Unlike the SFA, where the fluid can be assumed to be confined between two infinite parallel plates by invoking the Derjaguin approximation, atomic force microscopy (AFM) involves the interaction between a tip and a flat substrate. AFM experiments carried out with dodecane and octameth- 
yltetracyloxane (OMCTS) as the confining fluids, showed that layering and solvation force oscillations were not restricted to the SFA but must be present in the fluid confined between the tip and the substrate in AFM experiments. ${ }^{21,22}$ More recent experiments with enhanced resolution show that the magnitude of the forces observed in the AFM experiment are similar to those observed in the SFA. ${ }^{23,24}$ AFM experiments with carbon nanotube tips, where the contact area is well-defined, show the presence of well-defined oscillations in the solvation force response. ${ }^{25}$ These experiments reveal a very interesting character of the confined fluid; solvation forces occur over a wide range of areas of confinement and lead one to naturally question the existence of a minimum confinement area required for fluid layering and the resulting oscillatory solvation forces. Molecular dynamics simulations which seek to mimic the AFM experiment reveal the presence of solvation force oscillations when a fluid is confined between an atomically structured pyramidal tip or a sphere and a flat surface. ${ }^{26,27}$

A few molecular simulation studies have been used to probe the structure of fluids confined between confining surfaces which are rough on the nanoscale. The behavior of ultrathin monoatomic films have been investigated for a structured slit pore made up of one flat wall and the other corrugated with regularly spaced rectangular grooves. ${ }^{28}$ The registry of the walls is seen to play an important role on the equilibrium structures that form under confinement, and under certain conditions alternating strips of solid and fluid are seen to coexist in the pore. Grand canonical Monte Carlo (GCMC) simulations ${ }^{29-31}$ and density functional studies ${ }^{32}$ have been carried out to elucidate the role of surface roughness on solvation forces for monoatomic fluids confined in pores. In all of these studies, both the confining surfaces are assumed to be periodically rough. The effect of random roughness, studied using a tiled surface to obtain a distribution of pore widths, reveals a reduction in the solvation force oscillations with increasing roughness. ${ }^{29}$ A superposition approximation, wherein the solvation force response from the prototypical slit pore is used to predict the solvation force response for a fluid confined between rough surfaces, is accurate, provided the wavelength of the roughness is sufficiently large..$^{29,31}$ The superposition approximation is similar in spirit to the Derjaguin approximation and assumes that the solvation force response between two curved surfaces is similar to that between two infinitely flat surfaces. Apart from the work by Frink and von Swol, where the superposition approximation has been shown to be accurate for larger wavelengths of undulations, the range and validity of the superposition approximation for a given form of roughness has received little attention in the literature.

Given the large number of parameters and different possibilities for creating a rough surface, it is clear that a systematic analysis would entail studying the effects of the amplitude, degree of roughness, and distributions (in the case of randomly rough surfaces) on the local fluid structure. The parameter space is further expanded if both walls are rough. With the exception of the study by Curry et al. ${ }^{28}$ effects associated with structured walls have not been investigated. Since the magnitude of roughness is of the same order as the confined fluid diameter, it is likely that the three-dimensional fluid-wall potential associated with atomically structured pores might be important in influencing local density and structure in grooves or undulations that make up the rough surface.

In this paper, we investigate the influence of surface roughness on the solvation force, fluid structure, and thermodynamics of confined soft sphere fluids. In contrast to previous studies in rough pores, roughness is introduced via a sinusoidal undulation on one wall of the prototypical slit pore, while keeping the other flat. Two kinds of rough pores are studied. In the first case the fluid-wall interaction potential is a one-dimensional 10-4 potential where the interaction potential is only dependent on the normal fluid-wall distance. In the second case the rough wall is structured and hence made up of discrete atoms which interact with the fluid. By keeping one wall flat and varying the amplitude and wavelength of the undulations on the second wall, deviations in the density distributions, solvation force characteristics, and free energies from the slit-shaped pore can be systematically investigated. We illustrate that this choice of geometry allows one to phase shift the solvation force curve in a controlled manner. Grand potential calculations are used to examine the influence of structured walls and roughness on the thermodynamics of the confined fluid. In particular we investigate the influence of direct interactions between the pore walls on the free energy of the system. For both pore models we critically evaluate the accuracy of the superposition approximation in predicting the solvation force for fluids confined in rough pores. We finally discuss implications of our results on recent experimental observations on the effect of roughness in SFA experiments and the now well-established observation of solvation forces in AFM experiments.

\section{THEORY AND SIMULATION PROCEDURE}

\section{A. Rough pore models RPA and RPB}

Rough pores are constructed by introducing sinusoidal undulations on one wall of the prototypical slit pore. Two types of rough pores as illustrated in Figs. 1(a) and 1(b) have been studied. The rough pores are periodic in the $x$ and $y$ directions and characterized by a mean pore width $H$. All references to the pore width will refer to the mean pore width. We distinguish the pores by the nature of fluid-wall interactions used. In the first rough pore, henceforth referred to as RPA [Fig. 1(a)], the fluid-wall interaction potential is assumed to be the 10-4 potential,

$$
\psi(z)=2 \pi \epsilon_{f w}\left[\frac{2}{5}\left(\frac{\sigma_{f w}}{z}\right)^{10}-\left(\frac{\sigma_{f w}}{z}\right)^{4}\right]
$$

where $z$ [Fig. 1(a)] is the normal distance, measured from the wall, between the fluid particle and the wall, $\epsilon_{f w}$ is the fluidwall interaction parameter, and $\sigma_{f w}$ is the interaction diameter. The total fluid-wall interaction potential for fluid particle, $i$, with $z$ coordinate $z_{i}$ is

$$
\psi_{f w}=\psi\left(H / 2+z_{i}\right)+\psi\left(H / 2-z_{i}\right),
$$




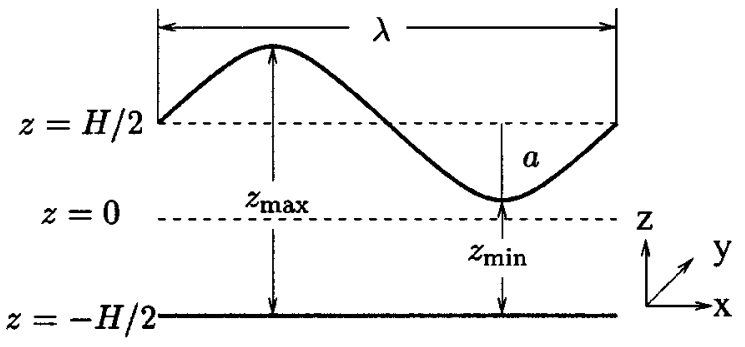

(a)

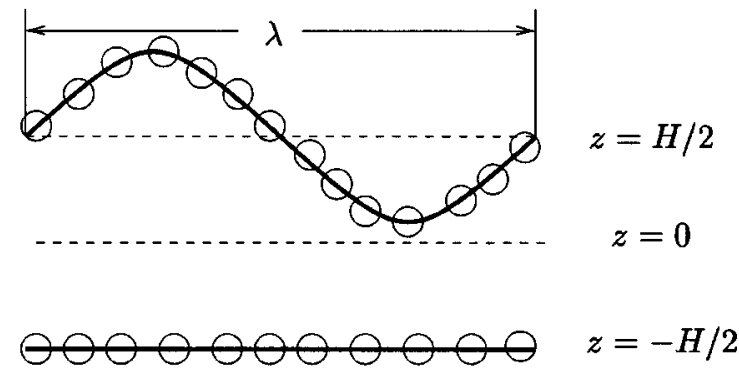

(b)

FIG. 1. Schematic of rough pores of mean pore width $H$ of (a) smooth walls and (b) structured walls, illustrating the wavelength and amplitude of the sinusoidal undulation in the wall located at a mean position $z=H / 2$.

where the first term represents contributions from the wall located at $z=-H / 2$ and the second term from the wall at $z$ $=H / 2$. The functional form for the coordinates of the sinusoidal wall at $z=H / 2$ is given by

$z(x)=a \sin \left(\frac{2 n \pi\left[x+L_{x} / 2\right]}{L_{x}}\right)+H / 2, \quad-L_{x} / 2 \leqslant x \leqslant L_{x} / 2$,

where $a$ is the amplitude of oscillation, $L_{x}$ is the simulation box length along the $x$ axis as shown in Fig. $1, L_{x} / n=\lambda$ is the wavelength of undulations in the top wall, and $H$ is the mean pore width. The degree of roughness is controlled by both the wavelength $\lambda$ and amplitude $a$.

In the second pore, henceforth referred to as RPB [Fig. 1(b)], the walls are made up of discrete atoms which interact with the pore fluid with a 12-6 Lennard-Jones (LJ) potential,

$$
\psi\left(r_{i j}\right)=4 \epsilon_{f w}\left[\left(\frac{\sigma_{f w}}{r_{i j}}\right)^{12}-\left(\frac{\sigma_{f w}}{r_{i j}}\right)^{6}\right],
$$

where $\psi\left(r_{i j}\right)$ is the interaction potential between a fluid particle $i$ and wall particle $j$ separated by a distance $r_{i j}$.

For the structured pore (RPB) atoms in the bottom wall $(z=-H / 2)$ were arranged in an hcp lattice, with lattice parameter $1.139 \sigma_{w w}$ corresponding to a surface density of $0.889 \sigma_{w w}^{-2}$. The $x$ and $y$ coordinates for the rough wall were similar to those of the bottom wall, however, the $z$ coordinate was evaluated using Eq. (3) for a given value of amplitude $a$ and wavelength $\lambda$. We will refer to both RPA and RPB as rough pores when $a \neq 0$ and for the cases where $a=0$ (flat surface) we will refer to them as slit pores. The $a^{*}=a / \sigma_{f f}$ values which were used for the simulations of rough pores are $0.25,0.5,1.0$, and 1.5 (RPA) with $14 \leqslant \lambda * \leqslant 2.33$. Fluid-
TABLE I. Reduced units. $\epsilon_{f f}$ and $\sigma_{f f}$ are the Lennard-Jones parameters for the fluid-fluid interactions.

\begin{tabular}{ll}
\hline \hline \multicolumn{1}{c}{ Quantity } & \multicolumn{1}{c}{ Reduced unit } \\
\hline Pore width & $H^{*}=H / \sigma_{f f}$ \\
Pore lengths & $L_{x}^{*}=L_{x} / \sigma_{f f}, L_{y}^{*}=L_{y} / \sigma_{f f}$ \\
Amplitude & $a^{*}=a / \sigma_{f f}$ \\
Wavelength & $\lambda^{*}=\lambda / \sigma_{f f}$ \\
Temperature & $T^{*}=k T / \epsilon_{f f}$ \\
Density & $\rho^{*}=\rho \sigma_{f f}^{3}$ \\
Solvation force & $f_{z}^{*}=f_{z} \sigma_{f f}^{3} / \epsilon_{f f}$ \\
Pressure (wall-wall) & $p_{w w}^{*}=p_{w w} \sigma_{f f}^{3} / \epsilon_{f f}$ \\
Excess grand potential & $\Delta \Omega_{\mathrm{ex}}^{*}=\Delta \Omega_{\mathrm{ex}} / \epsilon_{f f}$ \\
\hline \hline
\end{tabular}

fluid interactions are modeled using 12-6 LJ interactions with potential parameters $\sigma_{f f}$ and $\epsilon_{f f}$. For both RPA and RPB we have assumed that the fluid-fluid interaction parameters are similar to the fluid-wall interaction parameters. Hence $\epsilon_{f f}=\epsilon_{f w}$ and $\sigma_{f f}=\sigma_{f w}=\sigma_{w w}$.

\section{B. GCMC simulations}

We perform a series of GCMC simulations ${ }^{33}$ at different pore widths and various degrees of roughness. Since fluidfluid and fluid-wall interaction parameters are similar, simulations are conveniently carried out in reduced units (Table I). During a GCMC simulation, a pore of fixed volume, $V$, is equilibrated with a bulk fluid whose chemical potential $\mu$ and temperature $T$ are held fixed. The pore volumes for both RPA and RPB are based on the mean pore width $H$. Hence $V=H L_{x} L_{y}$ for both the slit and rough pores. Simulations typically consisted of $(10-15) \times 10^{6}$ equilibration moves followed by about $(10-15) \times 10^{6}$ moves during which system properties were averaged. Each Monte Carlo move consisted of an attempted particle insert, delete and displacement as per the standard Metropolis sampling procedure. ${ }^{33}$ The thermodynamic state of the bulk fluid corresponds to $T^{*}$ $\equiv k T / \epsilon_{f f}=1.2$ and $\rho^{*} \equiv \rho \sigma_{f f}^{3}=0.662$. At this state point the bulk pressure $P^{*}=P \sigma_{f f}^{3} / \epsilon_{f f}=0.399$ and activity $Z^{*}=Z \sigma_{f f}^{3}$ $=0.0751$. The activity $Z=\exp (\mu / k T) / \Lambda^{3}$ where the thermal de Broglie wavelength $\Lambda=h / \sqrt{2 \pi m k T} ; h$ is the Plancks constant and $m$ the mass of the particle. Simulations are carried out at fixed activity $Z$ which is equivalent to fixing the chemical potential $\mu$. The simulation box lengths used for pore RPA are $L_{x}=L_{y}=14 \sigma_{f f}$ and for RPB $L_{x}=13.81 \sigma_{f f}$ and $L_{y}=13.67 \sigma_{f f}$. In order to evaluate system size effects, simulations were carried out at smaller pore sizes $L_{x}=L_{y}=7 \sigma_{f f}$ in the case of RPA. Except for small quantitative differences at smaller pore widths the results were similar. Unless otherwise stated, all results correspond to those obtained from the larger system size. While interpreting our results it is useful to define two widths $z_{\min }$ and $z_{\max }$ as illustrated in Fig. 1 where $z_{\min }=H-a$ and $z_{\max }=H+a$.

\section{Density distributions}

We compute layer density distributions by dividing the pore into bins of width, $\Delta z=0.25 \sigma_{f f}$. The density distributions are evaluated using 


$$
\rho(z)=\frac{\left\langle N\left(z-\frac{\Delta z}{2}, z+\frac{\Delta z}{2}\right)\right\rangle}{V_{\Delta z}},
$$

where $\langle N(z-\Delta z / 2, z+\Delta z / 2)\rangle$ is the ensemble averaged number of atoms in $V_{\Delta z}$ which is the pore volume of a horizontal bin of thickness $\Delta z$ whose length and width correspond to the accessible region of the pore. Density distributions computed in this manner, although one-dimensional, enable the comparison of particle distributions between different rough pores and with the slit pore in a convenient manner.

\section{Thermodynamic relationships}

The thermodynamics of confined inhomogeneous fluids in smooth walled slit-shaped pores ${ }^{34-36}$ and for fluids between structured pores ${ }^{37}$ have been treated earlier in the literature. We extend the description to the rough pores geometries (Fig. 1) investigated in this study. The pore is in equilibrium with a bulk reservoir held at a fixed chemical potential $\mu$ and temperature $T$. $V$ represents the entire volume accessible to the fluid. The pores are held at a mean separation $H$ by applying a force of magnitude $F_{z}$ on each wall of the pore. The change in internal energy of the system is

$d U=-P_{b} d V+T d S+\mu d N-F_{z} d H+F_{x} d L_{x}+F_{y} d L_{y}$,

where $P_{b}$ is the bulk pressure, $S$ the entropy, $N$ the number of fluid particles, and $L_{x}$ and $L_{y}$ are the lateral dimensions of the pore in the $x$ and $y$ directions, respectively. The force $F_{z}$ has contributions from both the normal forces exerted by the inhomogeneous fluid confined within the pore and the forces due to the interaction of the two walls in vacuum. If $A_{x y}$ is the projected area in the $x-y$ plane then

$$
F_{z}=\left(\Pi+p_{w w}\right) A_{x y},
$$

where $\Pi=f_{z}-P_{b}$ is the disjoining pressure and $f_{z}$ is the solvation pressure also referred to in the literature (and in this paper) as the solvation force. The contributions to the solvation force are due to the interactions of the confined fluid with the walls of the pore. In Eq. (7), $p_{w w}$ represents the pressure due to the interactions between the two walls of the pore in vacuum. The interaction between the walls of the pores are assumed to be independent of the presence of the fluid molecules. The last two terms in Eq. (6) represent contributions to the changes in the internal energy arising from forces within the confined fluid, due to infinitesimal changes in the pore volume, brought about by altering the interfacial area by changing the lengths $L_{x}$ and $L_{y}$. Hence $F_{\alpha}$ in Eq. (6) $(\alpha=x, y)$ is the force in direction $\alpha$. In order to recast the last two terms in a more traditional representation involving changes in interfacial area, Eq. (6) can be rewritten as

$d U=-P_{b} d V+T d S+\mu d N-F_{z} d H+\gamma_{x} d A_{x}+\gamma_{y} d A_{y}$,

where $d A_{x}=L_{y} d L_{x}$ and $d A_{y}=L_{x} d L_{y}$ and $\gamma_{x}$ and $\gamma_{y}$ are the pore fluid interfacial tensions. Although the local pore height $z(x)$ is dependent on $L_{x}$ [Eq. (3)] stretching the plates by $d L_{x}$ is equivalent to an incremental change in the wavelength of the top plate, which by choice of the geometry of the top plate leaves the mean pore width $H$ unaltered. For the structured pore we assume that the wall atoms are always in perfect registry. From Eq. (8) the following relationships can be derived for the normal force:

$$
F_{z}=\left(\Pi+p_{w w}\right) A_{x y} \equiv-\left(\frac{\partial U}{\partial H}\right)_{V, S, N, A_{x}, A_{y}},
$$

and the interfacial tensions,

$$
\begin{aligned}
\gamma_{x} & =\left(\frac{\partial U}{\partial A_{x}}\right)_{V, S, N, H, A_{y}} \\
& \equiv \frac{1}{L_{x}} \int_{x=0}^{L_{x}} \int_{z=0}^{z(x)}\left[P_{b}-T_{x x}(x, z)\right] d z d x
\end{aligned}
$$

and

$$
\begin{aligned}
\gamma_{y} & =\left(\frac{\partial U}{\partial A_{y}}\right)_{V, S, N, H, A_{x}} \\
& \equiv \frac{1}{L_{x}} \int_{x=0}^{L_{x}} \int_{z=0}^{z(x)}\left[P_{b}-T_{y y}(x, z)\right] d z d x .
\end{aligned}
$$

The pore interfacial tensions $\gamma_{x}$ and $\gamma_{y}$ are obtained by averaging components of the corresponding stress tensor, $T_{\alpha \alpha}(x, z)$. For a pore of uniform width $z(x)=H$ the equations for the pore interfacial tension reduce to ${ }^{35}$

$$
\begin{aligned}
\gamma_{\alpha \alpha} & =\left(\frac{\partial U}{\partial A_{\alpha}}\right)_{V, S, N, H, A_{\beta}} \\
& \equiv \int_{z=0}^{H}\left[P_{b}-T_{\alpha \alpha}(z)\right] d z, \quad \alpha, \beta=x, y .
\end{aligned}
$$

Since the pore is in equilibrium with a bulk fluid, the appropriate potential is the grand potential

$$
\Omega=U-T S-\mu N .
$$

Using Eq. (8) the differential form for $\Omega$ is

$$
d \Omega=-P_{b} d V-S d T-N d \mu-F_{z} d H+\gamma_{x} d A_{x}+\gamma_{y} d A_{y} .
$$

The corresponding differential form of the grand potential for the bulk fluid (in the absence of the pore) is

$$
d \Omega_{b}=-P_{b} d V-S_{b} d T-N_{b} d \mu .
$$

Subtracting Eq. (15) from Eq. (14)

$d \Omega_{\mathrm{ex}}=-S_{\mathrm{ex}} d T-N_{\mathrm{ex}} d \mu-F_{z} d H+\gamma_{x} d A_{x}+\gamma_{y} d A_{y}$,

where $S_{\text {ex }}=S-S_{b}$ and $N_{\text {ex }}=N-N_{b}$. From Eq. (16)

$$
F_{z}=\left(\Pi+p_{w w}\right) A_{x y} \equiv-\left(\frac{\partial \Omega_{\mathrm{ex}}}{\partial H}\right)_{T, \mu, A_{x}, A_{y}} .
$$

$\Omega_{\text {ex }}$ can be obtained by integrating Eq. (17) at fixed $T, \mu$, $A_{x}$, and $A_{y}$ resulting in

$$
\frac{\Delta \Omega_{\mathrm{ex}}}{A_{x y}}=\int_{H}^{H_{\infty}}\left(\Pi+p_{w w}\right) d H \quad \text { for } H>H_{c},
$$

where $\Delta \Omega_{\mathrm{ex}}=\Delta \Omega_{\mathrm{ex}}(H)-\Delta \Omega_{\mathrm{ex}}\left(H_{\infty}\right), H_{c}$ is the critical pore width below which the pore density $\rho_{p}=0$. Since the pore is no longer accessible to fluid particles from the bulk reservoir 
for $H<H_{c}$, the above thermodynamic analysis which is developed for the confined fluid is valid only for $H>H_{c}$. While carrying out a GCMC simulation the pore walls are kept at a fixed distance $H$ and the disjoining pressure $\Pi$ is computed as a function of $H$. Hence $\Delta \Omega_{\mathrm{ex}}$ can be directly evaluated from Eq. (18).

The 10-4 fluid-wall potential [Eq. (1)] is derived for a particle interacting with a single layer of wall atoms with surface density $\rho_{w}=1.0 \sigma_{w w}^{-2}$. Hence the expression for the pressure between two walls, separated by a distance $H$ in vacuum, is

$$
p_{w w}=\frac{8 \pi \epsilon_{f w} \rho_{w}}{H}\left[\left(\frac{\sigma_{f w}}{H}\right)^{10}-\left(\frac{\sigma_{f w}}{H}\right)^{4}\right] .
$$

In the case of RPA, $p_{w w}$ is computed using Eq. (19) for the slit pore $\left(a^{*}=0\right)$ and a superposition principle is used to evaluate $p_{w w}$ for rough pores at different amplitudes. In the superposition principle, the pressure at a given local height in the rough pore is assumed to be similar to that predicted by Eq. (19). Hence $p_{w w}$ for the rough pores (RPA) is obtained by superposing the pressure contributions from the distribution of heights that make up the rough pore. The superposition principle is discussed in greater detail later in the manuscript. In the case of the structured pore, $p_{w w}$ is obtained by a direct summation of the normal forces exerted by the particles on the two walls, in vacuum, for different values of the slit width $H$.

The expression for evaluating the solvation force based on the fluid-wall interaction for the 10-4 potential, RPA, is

$$
f_{z}=-\frac{1}{2 A_{x y}} \sum_{i=1}^{N}\left\langle\frac{d \psi\left(H / 2+z_{i}\right)}{d z_{i}}+\frac{d \psi\left(H / 2-z_{i}\right)}{d z_{i}}\right\rangle,
$$

where $N$ is the number of fluid particles within the pore. The angular brackets represent an ensemble average. For RPB the normal force exerted by a fluid particle with the wall is computed using the 12-6 LJ potential [Eq. (4)]. In this paper, the solvation force is computed directly by evaluating Eq. (20) during a GCMC simulation.

\section{RESULTS AND DISCUSSION}

\section{A. Solvation force: RPA}

Figure 2 illustrates the solvation force as a function of mean pore width $H^{*}$ for fluid in RPA for different values of $a^{*}$ and $\lambda^{*}$. In all cases the solvation force for the slit pore $\left(a^{*}=0\right)$ has been plotted for comparison. At $a^{*}=0.25$ [Fig. 2(a) ] we find an overall decrease in the intensity and a broadening in the solvation force curves. Maxima and minima in the solvation force curves, although slightly phase shifted, occur at nearly similar pore widths at which maxima and minima occur in the slit pore. The phase shift is more apparent at smaller pore widths, where only a single fluid layer is accommodated in the pore, and seen to be shifted toward larger pore widths, when compared with the slit pore. At $a^{*}=0.25$ the solvation force values are relatively insensitive to the value of $\lambda^{*}$. The monotonic rise in the solvation force which is observed in the slit pore for $H^{*} \leqslant 2.25$ is absent and the solvation force is seen to decrease for $H^{*} \leqslant 2$. This is not
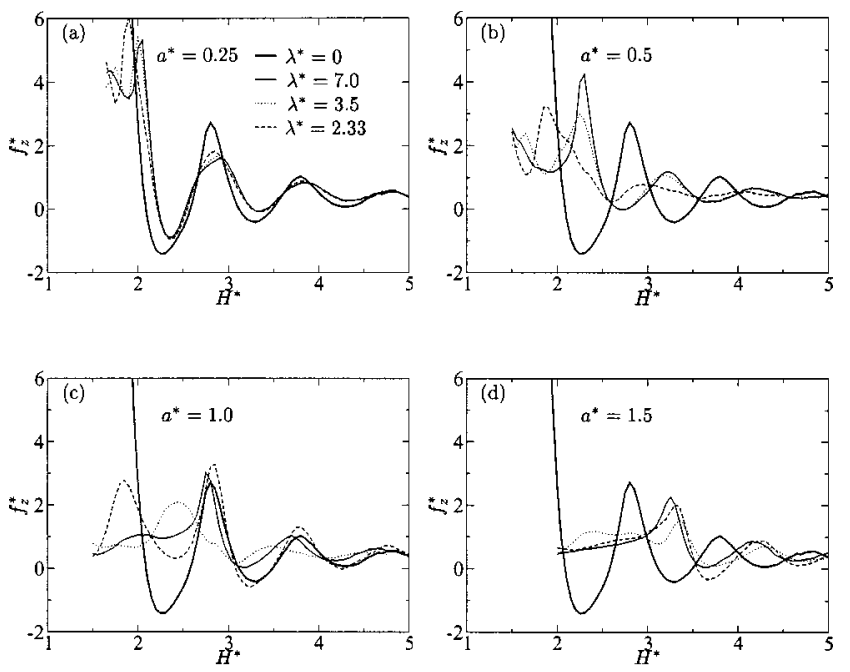

FIG. 2. Solvation force vs pore width for smooth pore RPA at different amplitudes, illustrating the influence of $\lambda^{*}$ on the solvation force curve. In all cases the solvation force curves are compared with the slit pore $\left(\lambda^{*}\right.$ $=0$ ). At $a^{*}=0.5$ and 1.5 the force curves are phase shifted by $0.5 \sigma$ when compared with the slit pore.

only due to the reduced volume of accessible pore space in the rough pore but primarily due to the ability of a small degree of roughness to disrupt the formation of a welldefined layer at the smaller pore widths.

At $a^{*}=0.5$ an interesting effect is observed. A phase shift of $0.5 \sigma_{f f}$ is observed in the solvation force curve [Fig. $2(b)]$ for all values of $\lambda^{*}$ excepting $\lambda^{*}=2.33$. Thus maxima and minima are reversed when compared with the slit pore. The strong repulsive regime in the solvation force at small pore widths for the slit pore is virtually absent for pore widths below $H^{*}=2.0$. At $a^{*}=1.0$ [Fig. 2(c)] with the exception of $\lambda^{*}=3.5$, the oscillations in the solvation force curves for the rough pores coincide approximately with the oscillations for the slit pore. The solvation force curves show similar trends for all the different wavelengths at larger pore widths. However, for smaller pore widths no systematic trends are observed. At $a^{*}=1.5$ [Fig. 2(d)] the oscillations in the solvation force start at larger pore widths when compared to the lower amplitude rough pores. Similar to the solvation force for $a^{*}=0.5$ [Fig. 2(b)] a phase shift of $0.5 \sigma_{f f}$ is observed. A broad nearly flat region is observed for $H^{*}<3.0$ and the damping in the force curve was found to be the greatest among all the different amplitudes of roughness.

Although the solvation force curves were phase shifted at a given amplitude of roughness, deviations in this general trend were observed at certain values of $\lambda^{*}$. These situations occurred at $a^{*}=0.5$ and $\lambda^{*}=2.33, a^{*}=1.0$ and $\lambda^{*}=3.5$, and $a^{*}=1.5$ and $\lambda^{*}=3.5$. At these conditions we ruled out the possibility of system size effects by carrying out simulations at $L_{x}^{*}=7$ and found that the results were unaltered. We return to the reasons for these deviations later in the text. In order to assess the sensitivity of the force curves to $\lambda^{*}$, we also carried out a few simulations at $\lambda *=14$ for $a^{*}=0.5$ and 1.0. The solvation force curves were identical to those re- 

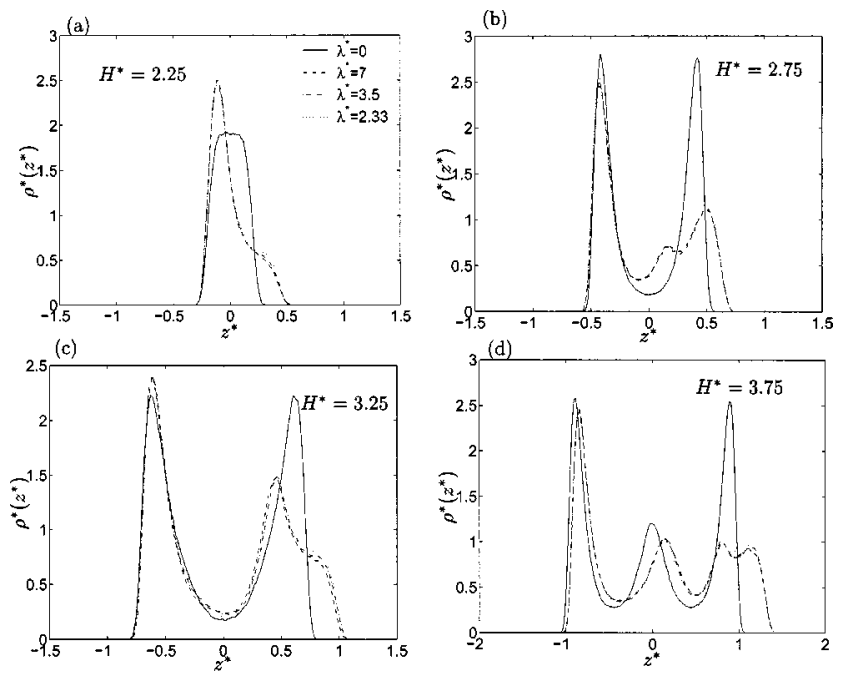

FIG. 3. Density distributions for RPA at $a^{*}=0.25$ as a function of $\lambda^{*}$. $H^{*}=2.25$ and 3.25 correspond to pore widths at which the solvation force is a local minima in the slit pore and $H^{*}=2.75$ and 3.75 correspond to local maxima in the solvation force curve (Fig. 2). The density distributions correspond to simulations carried out with box lengths, $L_{x}=L_{y}=7.0 \sigma_{f f}$

ported for $\lambda *=7.0$ indicating that the deviations in the solvation force curves occur only at smaller values of $\lambda^{*}$.

\section{B. Density distributions: RPA}

The density distributions corresponding to pore widths at which maxima and minima are observed in the solvation force for RPA with $a^{*}=0.25$ are shown in Fig. 3. The density distributions are relatively insensitive to the wavelength of roughness. A well-defined density peak near the flat wall at $z^{*}=-H^{*} / 2$ was observed in all cases indicating that a small roughness $\left(a^{*}=0.25\right)$ on the top wall is unable to disrupt layering at the bottom wall. At $H^{*}=2.25$ and 3.25 where the solvation force for the slit pore shows a minima [Fig. 2(a)], the density peak adjacent to the lower wall $\left(z^{*}\right.$ $\left.=-H^{*} / 2\right)$ for the rough pore [Figs. 3(a) and 3(c)] is of greater intensity than that for the slit pore, indicating improved layering in the fluid layer adjacent to the flat wall. As a consequence the solvation force at these two pore widths were found to be more repulsive [Fig. 2(a)] when compared with that of the slit pore. An opposite trend was observed at $H^{*}=2.75$ and 3.75 (maxima in $f_{z}^{*}$ for the slit pore) where significant disruption is observed in the layer adjacent to the rough wall, and layering at the bottom wall $\left(z^{*}=-H^{*} / 2\right)$ is reduced [Figs. 3(b) and 3(d)] when compared with the slit pore. This lowers the repulsion in the solvation force in the two layered regime for the rough pore [Fig. 2(a)].

At $a^{*}=0.5$, density distributions in the single layer regime [Fig. 4(a)] reveal increased ordering at the flat wall which, unlike the situation at $a^{*}=0.25$, improves as $\lambda^{*}$ is decreased. The increased amplitude leads to greater accessibility into the convex $z_{\max }$ regions of the pore. Although the peaks in density distributions at widths $H^{*}=2.25$ and $\lambda^{*}$ $=3.5$ and 2.33 indicate a greater degree of layering in the pore [Fig. 4(a)] the solvation force at these wavelengths are lower when compared to the largest wavelength, $\lambda *=7$ [Fig. 2(b)]. The reasons for these trends are discussed in subse-
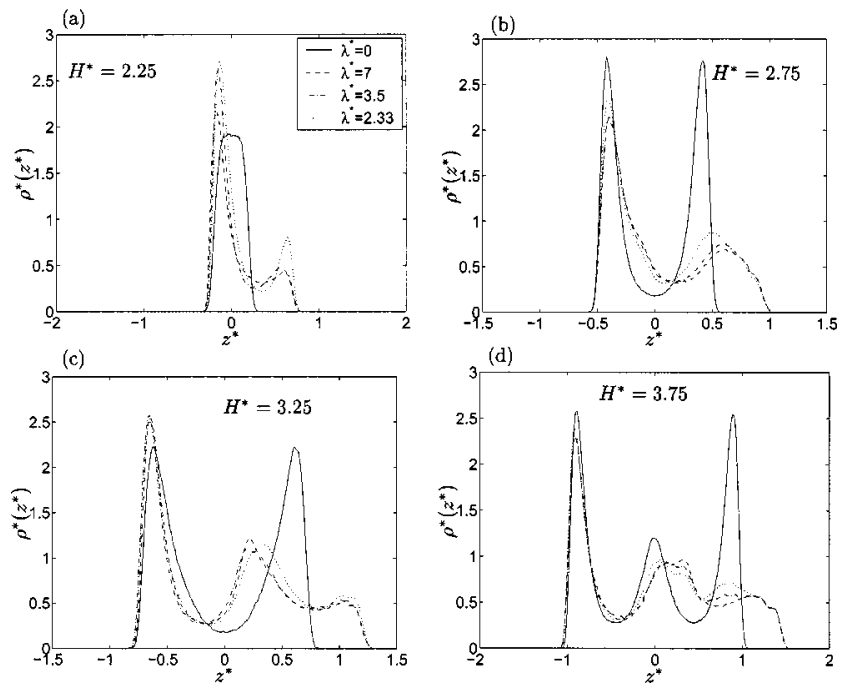

FIG. 4. Density distributions for RPA at $a^{*}=0.5$ as a function of $\lambda^{*} . H^{*}$ $=2.25$ and 3.25 correspond to pore widths at which the solvation force is a local minima in the slit pore and $H^{*}=2.75$ and 3.75 correspond to local maxima in the solvation force curve (Fig. 2). The density distributions correspond to simulations carried out with box lengths, $L_{x}=L_{y}=7.0 \sigma_{f f}$.

quent sections. At $H^{*}=3.25$ the splitting in the upper layer $\left(z^{*}=H^{*} / 2\right)$ is very definite, when compared with the corresponding situation at $a^{*}=0.25$ [Fig. 3(c)], giving rise to three layers as seen in the density distribution [Fig. 4(c)]. This is due to an increase in the number of particles in both the $z_{\min }$ and $z_{\max }$ regions of the pore.

\section{Superposition approximation: RPA}

In this section we evaluate the superposition approximation $^{29}$ to explain the observed phase shifts in the solvation force in RPA. We first present a qualitative explanation for the trends observed. As a representative case, we discuss the phase shift specifically for $a^{*}=0.5$. For $\lambda^{*}=7$ and 3.5 [Fig. 2(b)] the successive peaks in the solvation force occurred at $H^{*}=2.25$ and 3.25 and minima occurred at $H^{*}$ $=2.75$ and 3.75 , respectively. The phase shifts in the oscillations can be explained by comparing the value of the solvation force obtained in the slit pore $(n=0)$ at widths corresponding to $z_{\min }$ and $z_{\max }$ of the rough pore. At $H^{*}$ $=2.25, a^{*}=0.5, z_{\min }^{*}=1.75$ and $z_{\max }^{*}=2.75$. Since for the slit pore, $H^{*}=1.75$ and 2.75 correspond to a single layer and two layers, respectively, where the solvation force is positive (local maxima at $H^{*}=2.75$ ), the superposition of the positive solvation force at $z_{\min }$ and $z_{\max }$ results in a solvation force maxima at $H^{*}=2.25$. A similar effect, though reversed, is observed at $H^{*}=2.75\left(z_{\min }^{*}=2.25\right.$ and $z_{\max }^{*}$ $=3.25$ ) where the slit pore shows a local maximum in the solvation force and the rough pore produces a local minimum [Fig. 2(b)]. In contrast to the situation at $H^{*}=2.25$, here both $z_{\min }$ and $z_{\max }$ correspond to slit pore widths where the solvation force is a local minima, whose superposition results in a solvation force minimum for the rough pore. The phase shifts for the rough pore are consistent with the density distributions illustrated in Fig. 4, where increased layering is observed at $H^{*}=2.25$ and 3.25 and decreased layering at $H^{*}=2.75$ and 3.75 when compared with the slit pore. 

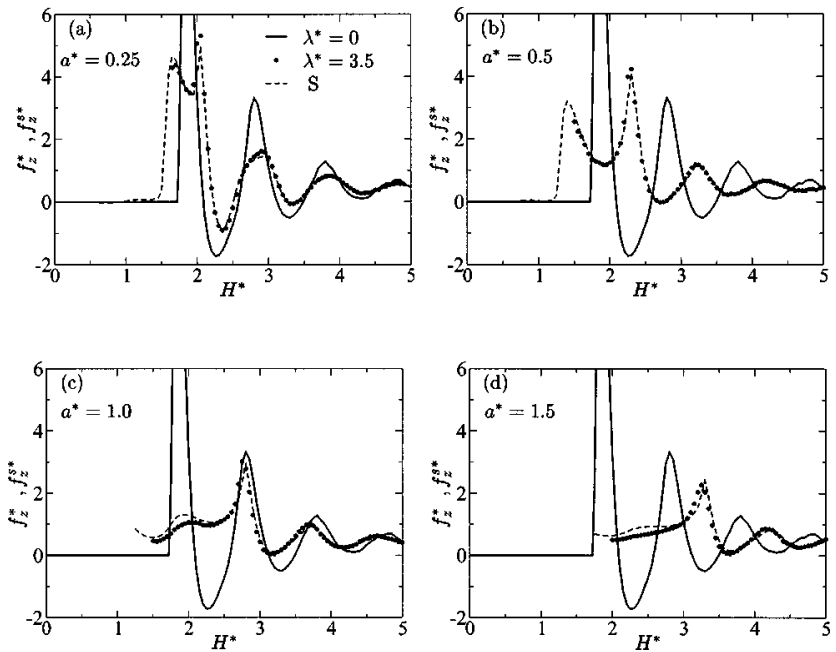

FIG. 5. Solvation forces $f_{z}^{s *}$ predicted using the superposition approximation (dashed lines) for RPA compared with simulation results (dots). The simulation results are shown for $\lambda *=7.0$ where the superposition approximation is seen to provide an excellent model for the solvation force.

We next apply the superposition approximation to predict the solvation force curves for the rough pores. ${ }^{29}$ The solvation force $f_{z}^{s}$ is obtained from the superposition approximation by assuming that the solvation force at a given location in the pore is similar to the solvation force observed in the slit pore whose width corresponds to the rough pore width $z(x)$ at that location. Hence

$$
f_{z}^{s}=\frac{1}{L_{x}} \int_{x=0}^{L_{x}} f_{z}[z(x)] d x \approx \frac{1}{M} \sum_{i=1}^{M} f_{z}\left[z\left(x_{i}\right)\right]
$$

We note that the superposition approximation is only a function of the overall distribution of widths and hence independent of the wavelength of roughness. ${ }^{29}$ The summation in Eq. (21) is carried out for $L_{x}=\lambda$ with $M=1000$. While using the superposition approximation, the solvation force curves $f_{z}$ for the slit pore was simulated until the pore was no longer able to admit particles. The results from using the superposition approximation [Eq. (21)] are shown in Fig. 5 where the solvation force curve for the slit pore is illustrated without the first maxima for better clarity. In all cases, $f_{z}^{s}$, obtained from the superposition approximation, was compared with the RPA results at $\lambda^{*}=7.0$. At this value of $\lambda^{*}$, the superposition approximation is seen to predict the solvation force for the rough surfaces for all the amplitudes considered extremely well. Even detailed variations in the simulated force curves at smaller pore widths are captured. Since the simulated force curves, at a fixed value of the amplitude, are not invariant with the wavelength of roughness (Fig. 2), the superposition approximation is seen to deteriorate for $\lambda^{*}$ $<7.0$. Since at larger wavelengths the local forces in the rough pores would be closer to those observed in a slit pore of similar width, based on the simulations at $\lambda^{*}=14$, our results suggest that the superposition approximation would be valid for rough pores where the wavelength $\lambda^{*}>7$ and $0.25<a^{*}<1.0$.
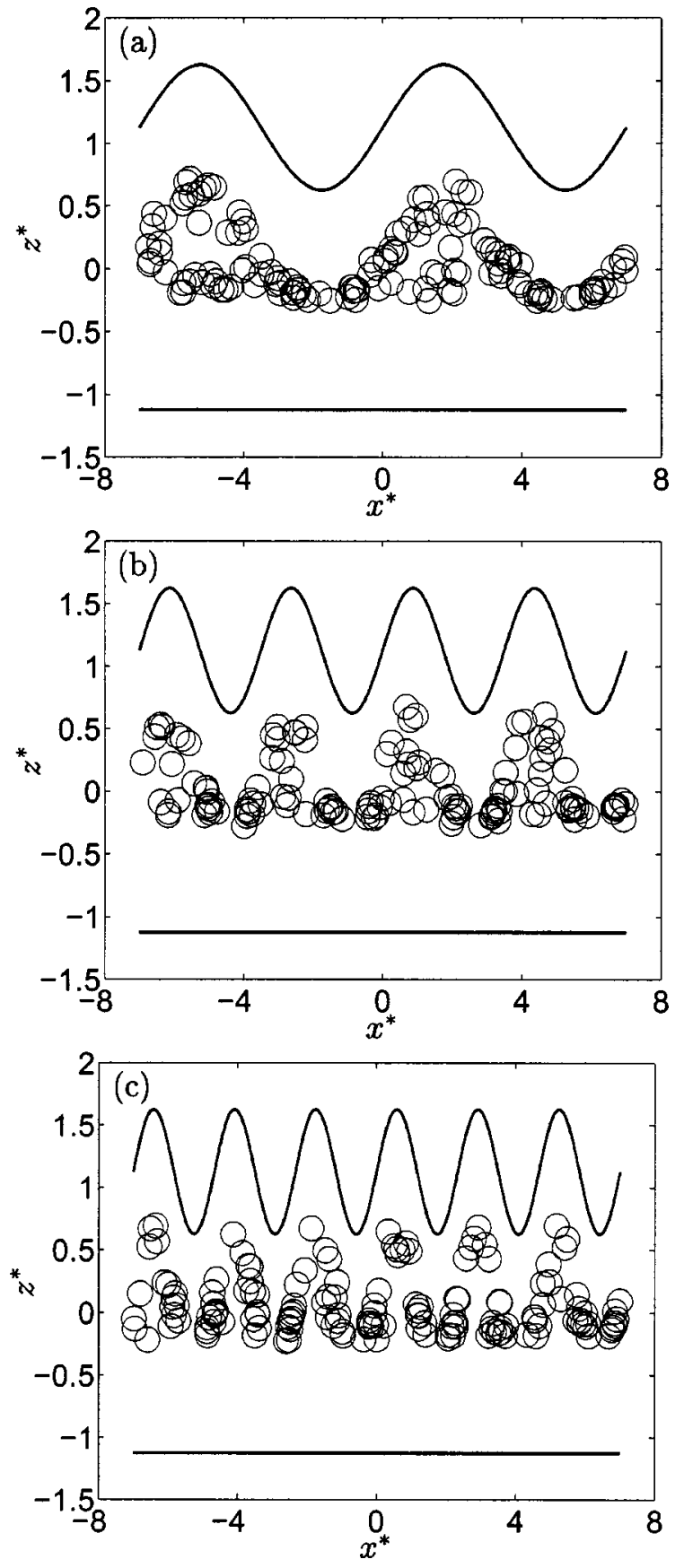

FIG. 6. Side-view snapshots for RPA at $H^{*}=2.25$ and $a^{*}=0.5$ as a function of $\lambda^{*}$. (a) $\lambda^{*}=7.0$, (b) $\lambda^{*}=3.5$, and (c) $\lambda^{*}=2.33$. Local layering is observed at the larger values of $\lambda^{*}$ (a) and (b). However at $\lambda^{*}=2.33$ (c) the fluid tends to align along the length of the undulations in the $y$ direction. This disrupts the ability of the fluid to form layers, leading to deviations in the solvation force trends as seen in Fig. 2(b) at this pore width.

\section{Deviations in solvation force: RPA}

We next investigate the reasons for the variations in the solvation force observed at a fixed value of $a^{*}$ and decreasing $\lambda^{*}$. For the superposition approximation to be valid, the locally averaged solvation force in the rough pore at a given value of $x$ should be similar to the solvation force in a slit pore at the same local pore width $[z(x)]$. As mentioned earlier in the text, deviations from this trend occur at smaller pore widths at particular values of $\lambda$ for $a^{*}=0.5,1.0$, and 

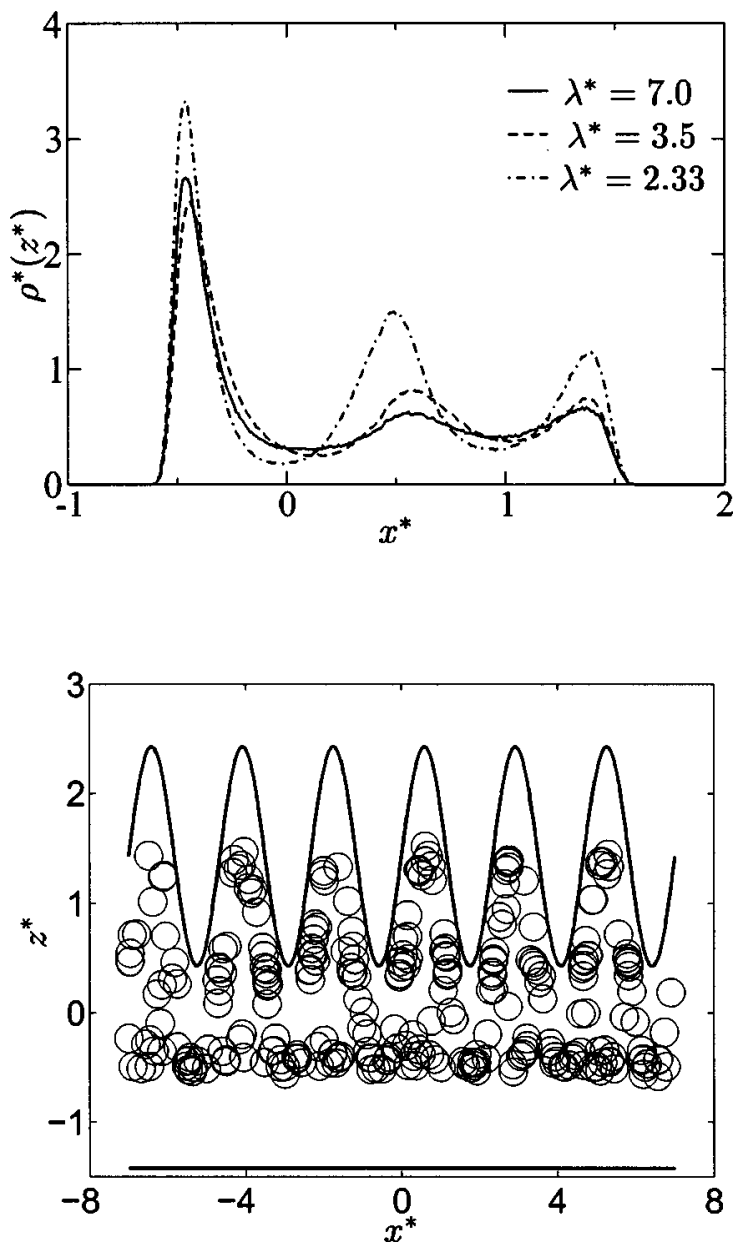

FIG. 7. Density distributions for RPA at $H^{*}=2.85$ and $a^{*}=1.0$ as a function of $\lambda^{*}$. The greatest degree of layering is observed at the smallest value of $\lambda *$. The bottom figure represents a snapshot of the $x-z$ configuration for $a^{*}=1.0$ where the formation of three distinct layers in the $z$ direction is clearly visible.

1.5. As a representative case we examine the situation at $a^{*}=0.5$ and $H^{*}=2.25$ where the phase shifted solvation force maxima observed at $H^{*}=2.25$ reduces in intensity with smaller $\lambda^{*}$ [Fig. 2(b)]. Side-view snapshots at $H^{*}$ $=2.25$ illustrated in Fig. 6 reveal that the ability of the fluid to form layers is gradually disrupted with decreasing wavelength. At the smallest value of $\lambda *=2.33$ [Fig. 6(c)] the fluid particles align in the $y$ direction, parallel to the undulations in the top wall. This alignment disrupts the ability of the fluid to form layers in the $z_{\min }$ and $z_{\max }$ regions destroying the superposition effect. Note that this preferential alignment of particles and not improved in-plane layering is responsible for the increased intensity in the density distribution as $\lambda^{*}$ is decreased [Fig. 4(a)]. A similar alignment of particles is responsible for the deviations in the solvation force at $a^{*}$ $=1.0$ and $\lambda^{*}=3.5$ [Fig. 2(c)] and $a^{*}=1.5$ and $\lambda^{*}=3.5$ [Fig. 2(d)]. The solvation force at $a^{*}=1.0$ and $\lambda^{*}=2.33$ does not follow the trend predicted by the superposition approximation, however, in this case, the phase shift is anomalously enhanced when compared to the large wavelengths and even gives rise to a peak at $H^{*}=1.8$ [Fig. 2(c)]. Examining the density distributions at $H^{*}=2.85$ [Fig. 7(a)] where a maxima occurs in the solvation force, the layering is par-
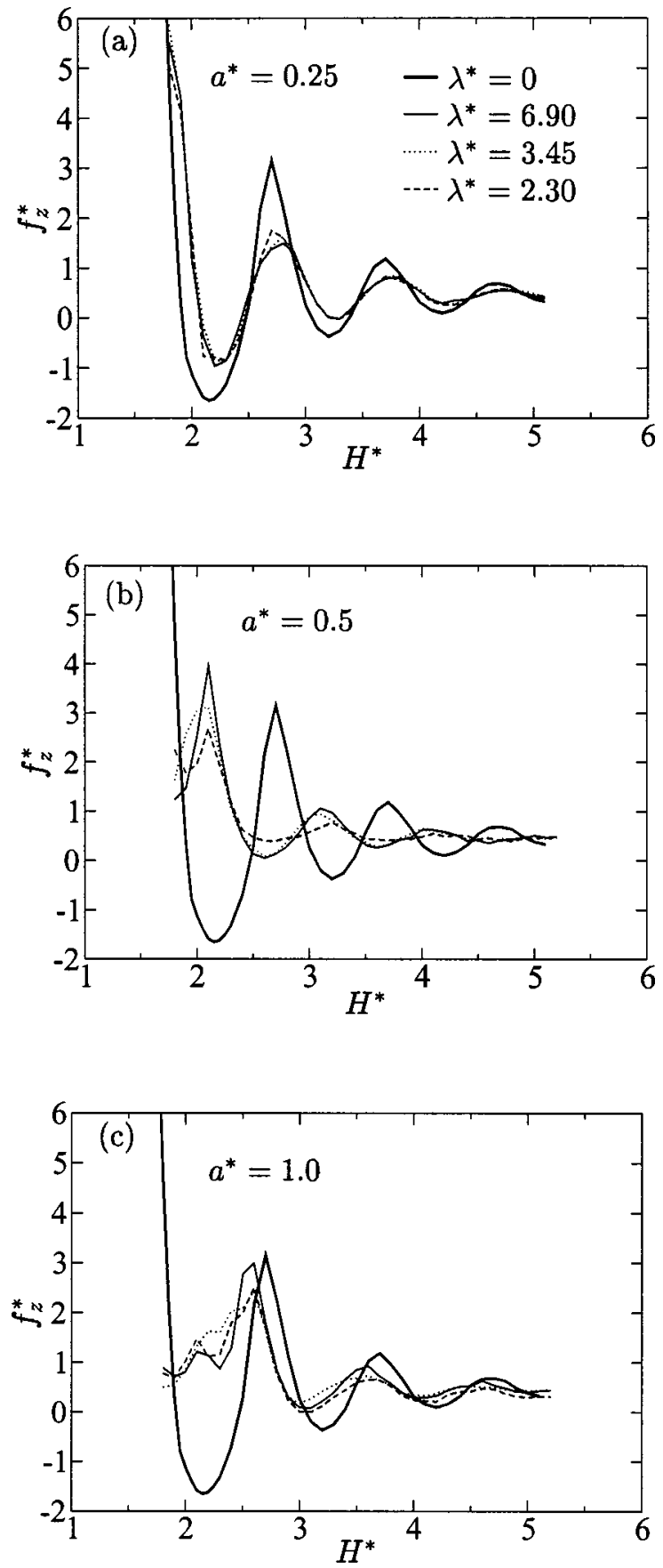

FIG. 8. Solvation force vs pore width for RPB. (a) $a^{*}=0.25$; (b) $a^{*}$ $=0.5$; and (c) $a^{*}=1.0$. For a fixed value of $a^{*}$, the degree of phase shift in the solvation force curve is uniform and relatively insensitive to $\lambda^{*}$. This is in contrast to the trends observed for RPA where the solvation force was sensitive to $\lambda^{*}$ particularly at larger amplitudes of roughness.

ticularly enhanced at $\lambda^{*}=2.33$. Side-view $(x-z)$ snapshots illustrated in Fig. 7(b) reveal this increased layering as $\lambda^{*}$ is decreased. The large amplitude and the one-dimensional nature of the fluid-wall potential contributes to this situation by enabling the fluid particles to access the $z_{\max }$ regions to a greater extent.

\section{E. Fluid in RPB}

The solvation force plots obtained from simulations for RPB are shown in Fig. 8. The primary difference in RPB is 
that the fluid-wall potential is a function of the $x, y$, and $z$ coordinates, whereas in RPA the potential was only a function of the $z$ coordinate. Since the qualitative features in the solvation force in RPB are similar to those observed in RPA only key differences will be discussed here. The most striking difference is the relative invariance in the solvation force curves to $\lambda^{*}$, as seen in Fig. 8. At $a^{*}=0.5$ [Fig. 8(b)] the phase shift occurs systematically for all values of $\lambda^{*}$ and unlike in the case of RPA the preferential alignment of particles along the $y$ axis at smaller $\lambda$ values was not observed. The solvation force oscillations and their relative insensitivity to changing $\lambda^{*}$ at a fixed value of $a^{*}$ are primarily due to the presence of discrete atoms on the walls giving rise to a more realistic three-dimensional fluid-wall interaction. The discrete nature of the walls in RPB reduces the entry into the $z_{\max }$ regions of the pore, preventing the alignment of the pore fluid along the $y$ axis. Note that this preferred alignment which occurs due to the one-dimensional nature of the fluidwall potential in RPA was solely responsible for the deviations from the expected trends.

Figure 9 illustrates the results of the superposition approximation for RPB. The results of the superposition approximation are compared with the results for $\lambda *=6.9$ for different amplitudes of roughness. As in the case of RPA the superposition approximation is seen to provide an excellent representation to the solvation force curves for the rough pores. The relative invariance in the solvation force curves for the wavelengths used in this study (Fig. 8) suggest that the superposition approximation has a greater range of validity for the structured pores than for the smooth walled pores.

The density distributions at $a^{*}=0.25$ [Figs. 10(a) and 10(b)] are relatively insensitive to $\lambda *$. However, in the vicinity of the rough wall the density distributions reveal that the accessibility into the $z_{\max }$ regions of the pore reduces with decreasing $\lambda^{*}$. This effect, though small, at $a^{*}=0.25$ was not observed in the case of RPA (Fig. 3). This decreased accessibility into the rough wall as $\lambda *$ is decreased is greater at $a^{*}=0.5$ [Figs. 10(c) and 10(d)]. The more realistic threedimensional fluid-wall interaction potential reduces the possibility of increased alignment of fluid particles in the $y$ direction. This difference in the density distributions between the two pore models is reflected in the relative insensitivity of the solvation force curve to the wavelength of roughness in the case of RPB.

\section{F. Grand potential: RPA and RPB}

The influence of roughness on the thermodynamics of the confined fluid is evaluated by computing the excess grand potential $\Delta \Omega_{\mathrm{ex}}^{*}$ as a function of the mean pore width $H$ using Eq. (18). The pore width at which the global minima in $\Delta \Omega_{\mathrm{ex}}^{*}$ occurs corresponds to the thermodynamically stable state of the confined fluid. In order to assess the influence of the normal pressure contribution due to interactions between the walls $\left(p_{w w}\right)$ of the slit pore we evaluate $\Delta \Omega_{\mathrm{ex}}^{*} / A_{x y}^{*}$ with and without the contribution from $p_{w w}$. Figure 11 illustrates $p_{w w}$ for both smooth and structured pores as a function of the slit width $H$ and amplitudes of roughness. The greatest differences in $p_{w w}$ between RPA and RPB are seen at smaller
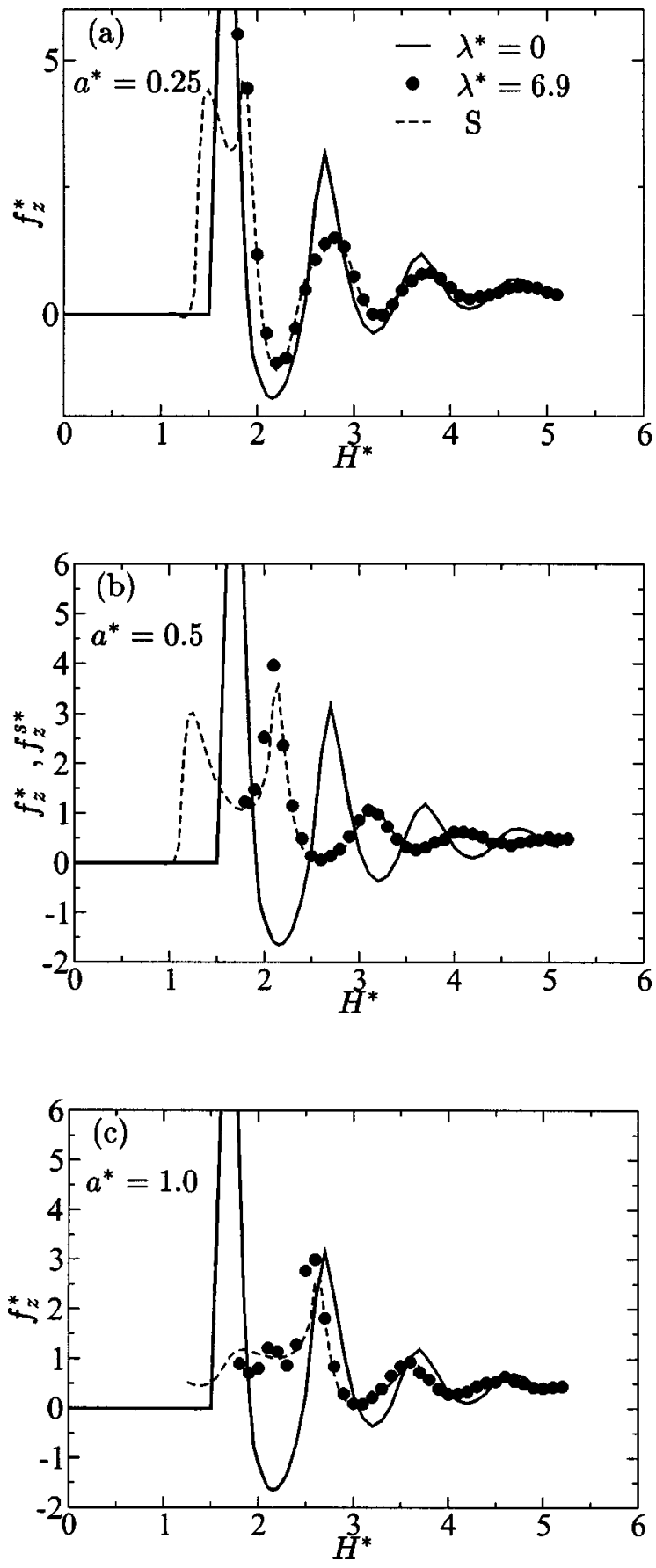

FIG. 9. Solvation forces $f_{z}^{s^{*}}$ predicted using the superposition (S) approximation (dashed lines) for RPB compared with simulation results (dots). The simulation results are shown for $\lambda^{*}=6.9$ where the superposition approximation is seen to provide an excellent model for the solvation force for RPB over a wide range of pore widths.

amplitudes in the vicinity of the minima in the force, where the lack of structure in the walls for RPA results in a greater attractive force between the walls. At a fixed amplitude, differences in the pressure contributions between the two types of walls decrease, as $H$ is increased. As a consequence the differences in pressure between the two walls reduces with increasing amplitude of roughness. The overall similarities between the pressure characteristics from RPA and RPB suggest that it is reasonable to use the superposition approxima- 

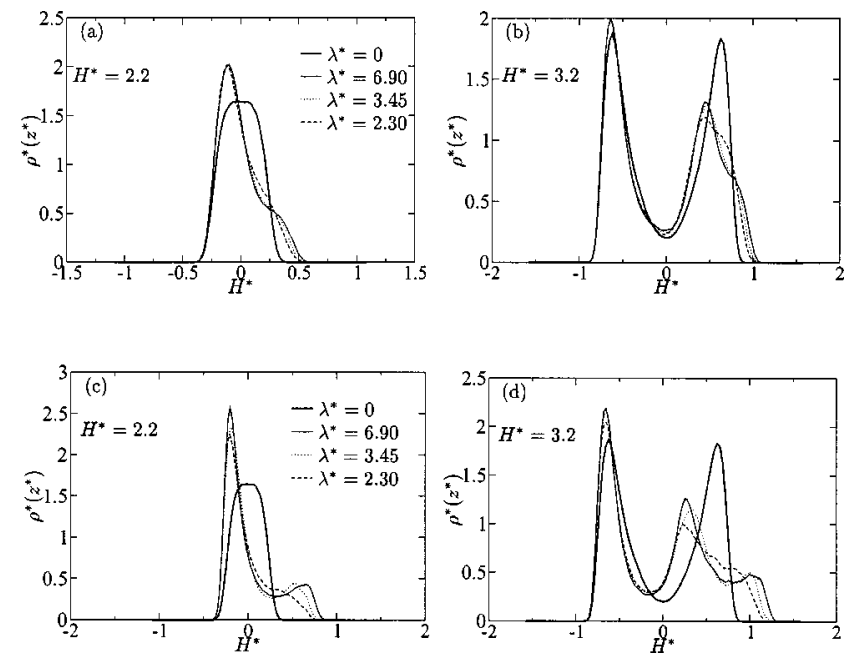

FIG. 10. Density distributions for RPB at $a^{*}=0.25$ (a) and (b) and at $a^{*}$ $=0.5$ (c) and (d). The lower accessibility into the $z_{\max }$ regions of the pore as the wavelength is decreased is clearly observed.

tion to compute the pressure contributions in the case of RPA. Note that the surface density of atoms in RPA is 1.0 $\sigma_{f f}^{-2}$ and in the case of RPB is $0.889 \sigma_{f f}^{-2}$.

Figure 12 illustrates $\Delta \Omega_{\mathrm{ex}}^{*} / A_{x y}^{*}$ for RPA and RPB for different amplitudes of roughness. For clarity, the data without wall-wall contributions $\left(p_{w w}=0\right)$ have been shifted by two positive $y$-axis units. The wavelengths of roughness correspond to $\lambda^{*}=7.0$ and 6.9 for RPA and RPB, respectively. For the slit pores $\left(a^{*}=0\right)$ the disjoining pressure obtained from GCMC simulations were used to compute $\Delta \Omega_{\mathrm{ex}}^{*} / A_{x y}^{*}$. For nonzero amplitudes the disjoining pressures were obtained using the superposition approximation. For the slit pore [Fig. 12(a)] the global minima in $\Delta \Omega_{\mathrm{ex}}^{*} / A_{x y}^{*}$ occurs at $H^{*}=2.07$ for RPA in the absence of wall-wall interactions. This corresponds to a single fluid layer in the pore. The presence of wall-wall interactions shifts the minimum to $H^{*}=2.05$ for $H^{*}>H_{c}^{*}$. Similarly in the case of RPB, a global minima in the absence of wall-wall interactions occurs at $H^{*}=1.9$ which shifts to $H^{*}=1.87$ in the presence of

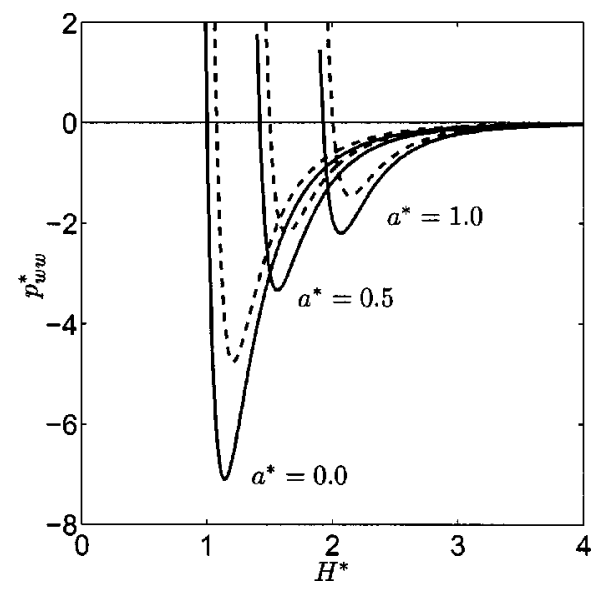

FIG. 11. Reduced normal pressure contribution between two surfaces of the slit pore for RPA (full lines) and RPB (dashed lines) for different amplitudes of roughness. The differences between RPA and RPB decrease as the amplitude of roughness increases.
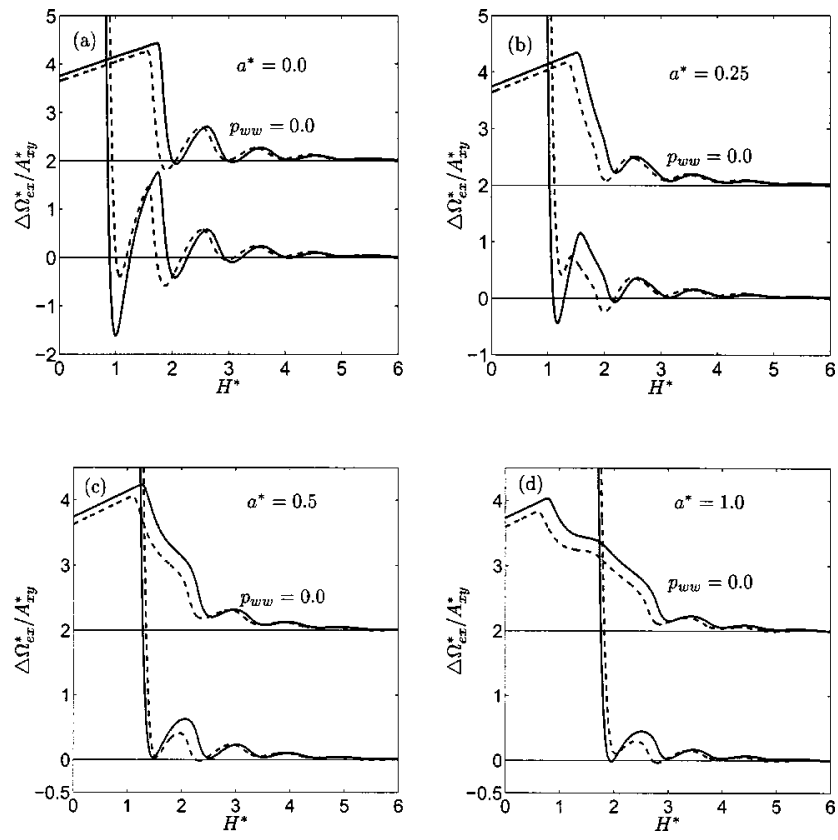

FIG. 12. Reduced excess grand potential per unit area for different values of the amplitude of roughness. The solid lines correspond to RPA and dashed lines to RPB. For better clarity, the data in the absence of wall-wall interactions $\left(p_{w w}\right)$ have been shifted by two positive $y$-axis units. The presence of wall-wall interactions creates a global minimum in the grand potential at finite pore widths as the amplitude of roughness is increased. For $a^{*}=0.5$, 1.0 , the first minimum in the grand potential occurs at pore widths, $H$ $>H_{c}$ where $H_{c}$ is the pore width at which the pore is inaccessible to the fluid.

wall-wall interactions. At these pore widths, the pore fluid is found to epitaxially freeze in the case of RPB. At $a^{*}=0.25$ [Fig. 12(b)], $\Delta \Omega_{\mathrm{ex}}^{*} / A_{x y}^{*}$ does not reveal the presence of a stable configuration at finite pore widths in the absence of wall-wall interactions. In the presence of wall-wall interactions, however, minima are located at $H^{*}=2.18$ and 2.0 for RPA and RPB, respectively, for $H^{*}>H_{c}^{*}$. At $a^{*}=0$ and 0.25 , the critical pore width, $H_{c}^{*}$, below which fluid particles can no longer access the pore is determined from the pore width below which the solvation force is zero. The first minima in $\Delta \Omega_{\mathrm{ex}}^{*} / A_{x y}^{*}$ which occurs for $H<H_{c}^{*}$ at $a^{*}=0$ and $a^{*}=0.25$ is purely due to the attractive wall-wall contributions as fluid particles are completely excluded from the system in this region. Hence minima occurring in the region $H^{*}<H_{c}^{*}$ are not included in the search for the equilibrium state of the system.

In the case of $a^{*}=0.5$ [Fig. 12(c)] and $a^{*}=1.0$ [Fig. $12(d)]$ the trends are qualitatively similar to those observed at smaller amplitudes of roughness, however, the first minima in $\Delta \Omega_{\mathrm{ex}}^{*} / A_{x y}^{*}$ occurred at pore widths where $H^{*}$ $>H_{c}^{*}$ and hence must be included in the search for the global minima. For $a^{*}=0.5$, global minima in $\Delta \Omega_{\mathrm{ex}}^{*} / A_{x y}^{*}$ were found to occur at $H^{*}=2.32$ for RPB when wall-wall contributions were included. For RPA, wall-wall contributions did not result in a global minima at finite pore widths. In the case of $a^{*}=1.0$ the minima were located at 1.95 and 2.8 for RPA and RPB, respectively, when wall-wall interactions were present. We did not extend the simulations to determine $H_{c}^{*}$ for $a^{*}=0.5$ and 1.0, however, simulations were carried 
out to ensure that the pore was accessible to the fluid for pore widths below the $H^{*}$ corresponding to the first minima in the excess grand potential.

The results in the absence of wall-wall interactions are similar to those obtained in an earlier study ${ }^{29}$ where increasing roughness is seen to thermodynamically favor a thicker fluid film. However, our study reveals that wall-wall interactions play a role at smaller pore widths as the degree of roughness is increased and should be included while searching for the true global minimum in the free energy. If $\Pi$ $\gg p_{w w}$ wall-wall interactions will play a minor role in deciding the thermodynamically stable pore width. Whether wall-wall interactions can be neglected would depend on both the strength of the fluid-wall interaction and the state of the bulk fluid. Since the primary goal of this work was to compare confined fluid behavior between smooth and rough pore models we have restricted our study to the 10-4 fluidwall potential which represents a weakly attractive wall. Before concluding we point out that the free energy analysis for the rough pores has been carried out keeping both surfaces in perfect registry. Previous simulations in rough pores (with one-dimensional fluid-wall potentials similar to RPA) suggest that although the global minima is found at perfect registry the approach to the final equilibrium state could involve configurations that are not in registry. ${ }^{29}$

\section{SUMMARY AND CONCLUSIONS}

We have carried out GCMC simulations to study the solvation force and density distributions for a fluid confined in a rough pore. Unlike previous studies, rough pores were constructed by taking a slit pore and introducing sinusoidal undulations on the top wall. Roughness was varied by changing the amplitude and wavelength of the undulation. Two kinds of rough pores were investigated. In RPA a 10-4 potential is used to model the fluid-wall interaction, where the interaction is only a function of the normal fluid-wall distance. In RPB the walls are made up of discrete atoms which interact with the fluid via a 12-6 LJ potential. For both RPA and RPB, the fluid-fluid interactions were modeled using a 12-6 LJ potential. In all cases results were compared and contrasted with fluid behavior in slit-shaped pores.

Our study reveals that by introducing sinusoidal roughness on one wall of the prototypical slit pore, solvation force curves can be phase shifted in a systematic manner. The extent of the phase shift can be controlled by altering the amplitude of the undulation. Comparisons between RPA and RPB indicate that the one-dimensional fluid-wall interaction potential in the case of RPA can give rise to force curves that deviate from expected trends. These deviations are caused by an increased sensitivity of the solvation force to the wavelength of the undulation at a fixed amplitude which arises due to increased accessibility of fluid particles in the $z_{\max }$ regions of the pore. However, for sufficiently large wavelengths $\left(\lambda^{*} \geqslant 7.0\right)$ our study suggests that the smooth-wall approximation is adequate. In the case of RPB, at a fixed amplitude, the force curves were relatively insensitive to the wavelength of undulation. This was due to the more realistic three-dimensional nature of the fluid-wall interaction.
The reduction in the magnitude and range of oscillations even at small amplitudes of roughness reported here and in other simulation studies on rough surfaces ${ }^{29,31}$ are consistent with recent observations that nanoparticles ${ }^{19,20}$ (coverage of $<1 \%$ ) present on the mica surface decrease the range and magnitude of oscillations in SFA experiments. In addition, the present and previous study ${ }^{29}$ reveal that roughness results in a positive shift of the solvation force curve and offers a partial explanation for the increased repulsion (smaller attractive regions) in the force curves, observed in SFA experiments $^{2,38,39}$ with OMCTS. Grand potential calculations from the computed solvation force curves reveal that including direct interactions between the two walls can significantly alter the slit width corresponding to the thermodynamically stable state. The interaction between walls has a weaker influence for small amplitudes of roughness $\left(a^{*}\right.$ $<0.25$ ), playing a more significant role as the amplitude of roughness is increased.

We have evaluated the accuracy of the superposition principle which uses the solvation force curve from the slit pore to predict the solvation force for the rough pores. In the case of RPA the superposition approximation works better for larger wavelengths and is able to predict the simulated results very accurately. In the case of structured pores, due to the relatively small variation in the solvation force as a function of the wavelength of roughness, the superposition approximation is able to predict the solvation force curves for wavelengths of the undulation as small as $\lambda^{*}=2.3$. The ability of the superposition approximation to predict solvation forces for rough surfaces from the slit pore solvation force response implies the following. Normal forces exerted by a fluid confined between infinite parallel plates are similar to the local forces, beneath small wavelength undulations in a rough pore at corresponding pore widths. These results have a direct bearing on interpreting results from AFM experiments and their similarities with the force response obtained from SFA experiments. Our study indicates that it is quite natural to expect solvation forces arising from AFM experiments (tip-surface interactions) which are quantitatively similar to those observed in SFA (surface-surface interactions) experiments.

\section{ACKNOWLEDGMENTS}

One of us (K.G.A.) would like to thank Sanjay Biswas, K. S. Gandhi, and H. T. Davis for several insightful and encouraging discussions while this work was in progress.

${ }^{1}$ J. N. Israelachvili, Intermolecular and Surface Forces: With Applications to Colloidal and Biological Systems (Academic, London, 1985).

${ }^{2}$ J. Klein and E. Kumacheva, J. Chem. Phys. 108, 6996 (1998).

${ }^{3}$ L. A. Demirel and S. Granick, J. Chem. Phys. 115, 1498 (2001).

${ }^{4}$ M. Heuberger, M. Zäch, and N. D. Spencer, Science 292, 905 (2001).

${ }^{5}$ I. K. Snook and W. van Megen, J. Chem. Phys. 72, 2907 (1980).

${ }^{6}$ M. Schoen, D. J. Diestler, and J. H. Cushman, J. Chem. Phys. 87, 5464 (1987)

${ }^{7}$ S. A. Somers and H. T. Davis, J. Chem. Phys. 96, 5389 (1992).

${ }^{8}$ R. Radhakrishnan and K. E. Gubbins, Mol. Phys. 96, 1249 (1998).

${ }^{9}$ J. Gao, W. D. Luedtke, and U. Landman, Phys. Rev. Lett. 79, 705 (1997).

${ }^{10}$ P. Bordarier, B. Rousseau, and A. H. Fuchs, Mol. Simul. 17, 199 (1996).

${ }^{11}$ C. Ghatak and K. G. Ayappa, Phys. Rev. E 64, 051507 (2001).

${ }^{12}$ K. G. Ayappa and C. Ghatak, J. Chem. Phys. 117, 5373 (2002). 
${ }^{13}$ P. A. Thompson and M. O. Robbins, Phys. Rev. E 41, 6830 (1990).

${ }^{14}$ B. Bhushan, J. N. Israelachvilli, and U. Landman, Nature (London) 374, 607 (1995).

${ }^{15}$ R. A. Quon, A. Ulman, and T. K. Vanderlick, Langmuir 16, 8912 (2000).

${ }^{16}$ J. B. Sweeney, H. T. Davis, and L. E. Scriven, Langmuir 9, 1551 (1993).

${ }^{17}$ B. Bhushan, Wear 225, 465 (1999).

${ }^{18}$ H. K. Christenson, J. Phys. Chem. 90, 4 (1986).

${ }^{19}$ M. Heuberger and M. Zäch, Langmuir 19, 1943 (2003).

${ }^{20}$ Y. Zhu and S. Granick, Langmuir 19, 8148 (2003).

${ }^{21}$ J. O'Shea, M. E. Welland, and T. Rayment, Appl. Phys. Lett. 60, 2356 (1992).

${ }^{22}$ J. O'Shea, M. E. Welland, and J. B. Pethica, Chem. Phys. Lett. 223, 336 (1994).

${ }^{23}$ R. Lim, S. F. Y. Li, and S. J. O’Shea, Langmuir 18, 6116 (2002).

${ }^{24}$ R. Lim and S. J. O'Shea, Phys. Rev. Lett. 88, 246101 (2002).

${ }^{25}$ S. P. Jarvis, in Nano-Surface Chemistry, edited by M. Rosoff (Marcel Dekker, New York, 2001), Chap. 2, pp. 17-58.

${ }^{26}$ D. L. Patrick and R. M. Lynden-Bell, Surf. Sci. 380, 224 (1997).

${ }^{27}$ L. D. Gelb and R. M. Lynden-Bell, Phys. Rev. B 49, 2058 (1994).
${ }^{28}$ J. E. Curry, F. Zhang, J. H. Cushman, M. Schoen, and D. J. Diestler, J. Chem. Phys. 101, 10824 (1994).

${ }^{29}$ L. J. D. Frink and F. van Swol, J. Chem. Phys. 108, 5588 (1998).

${ }^{30}$ D. J. Diestler and M. Schoen, Phys. Rev. E 62, 6615 (2000).

${ }^{31}$ F. Porcheron, M. Schoen, and A. H. Fuchs, J. Chem. Phys. 116, 5816 (2002).

${ }^{32}$ D. Henderson, S. Sokolowski, and D. Wasan, Phys. Rev. E 57, 5539 (1998).

${ }^{33}$ M. P. Allen and D. J. Tildesley, Computer Simulations of Liquids, 1st ed. (Clarendon, Oxford, UK, 1987).

${ }^{34}$ S. G. Ash, D. H. Everett, and C. Radke, J. Chem. Soc., Faraday Trans. 2 69, 1256 (1973).

${ }^{35}$ J. J. Magda, M. Tirrel, and H. T. Davis, J. Chem. Phys. 83, 1888 (1985).

${ }^{36}$ R. Evans and U. M. B. Marconi, J. Chem. Phys. 86, 7138 (1987).

${ }^{37}$ D. J. Diestler, M. Schoen, J. E. Curry, and J. H. Cushman, J. Chem. Phys. 100, 9140 (1994).

${ }^{38}$ H. K. Christenson, J. Chem. Phys. 78, 6906 (1983).

${ }^{39}$ P. Attard and J. L. Parker, J. Phys. Chem. 96, 5086 (1992). 